\title{
Not all brawn, but some brain. Strength gains after training alters kinematic motor abundance in hopping
}

\author{
Bernard X.W. Liew ${ }^{\text {Corresp.. }}{ }^{1}$, Andrew Morrison ${ }^{2}$, Hiroaki Hobara ${ }^{3}$, Susan Morris ${ }^{4}$, Kevin Netto ${ }^{4}$ \\ ${ }^{1}$ Centre of Precision Rehabilitation for Spinal Pain (CPR Spine), School of Sport, Exercise and Rehabilitation Sciences, College of Life and Environmental \\ Sciences, University of Birmingham, Birmingham, United Kingdom \\ 2 Sport and Exercise Sciences, Faculty of Science and technology, Anglia Ruskin University, Cambridge, United Kingdom \\ 3 Human Informatics Research Institute, National Institute of Advanced Industrial Science and Technology (AIST), Tokyo, Japan \\ ${ }^{4}$ School of Physiotherapy and Exercise Sciences, Curtin University of Technology, Perth, Western Australia, Australia
}

Corresponding Author: Bernard X.W. Liew

Email address: LiewB@adf.bham.ac.uk

Background. The effects of resistance training on a muscle's neural, architectural, and mechanical properties are well established. However, whether resistance training can positively change the coordination of multiple motor elements in the control of a well-defined lower limb motor performance objective remains unclear. Such knowledge is critical given that resistance training is an essential and ubiquitous component in gait rehabilitation. This study aimed to investigate if strength gains of the ankle and knee extensors after resistance training increases kinematic motor abundance in hopping.

Methods. The data presented in this study represents the pooled group results of a sub-study from a larger project investigating the effects of resistance training on load carriage running energetics. 30 healthy adults performed self-paced unilateral hopping, and strength testing before and after six weeks of lower limb resistance training. Motion capture was used to derive the elemental variables of planar segment angles of the foot, shank, thigh, and pelvis, and the performance variable of leg length. Uncontrolled manifold analysis (UCM) was used to provide an index of motor abundance (IMA) in the synergistic coordination of segment angles in the stabilization of leg length. Bayesian Functional Data Analysis was used for statistical inference, with a non-zero crossing of the $95 \%$ Credible Interval (Crl) used as a test of significance.

Results. Depending on the phase hop stance, there were significant main effects of ankle and knee strength on IMA, and a significant ankle by knee interaction effect. For example at $10 \%$ hop stance, a 1 $\mathrm{Nm} / \mathrm{kg}$ increase in ankle extensor strength increased IMA by 0.37 (95\% Crl 0.14 to 0.59), a $1 \mathrm{Nm} / \mathrm{kg}$ increase in knee extensor strength decreased IMA by 0.29 (95\% $\mathrm{Crl} 0.08$ to 0.51 ), but increased the effect of ankle strength on IMA by 0.71 (95\% Crl 0.10 to 1.33). At $55 \%$ hop stance, a $1 \mathrm{Nm} / \mathrm{kg}$ increase in knee extensor strength increase IMA by 0.24 (95\% $\mathrm{Crl} 0.001$ to 0.48 ), but reduced the effect of ankle strength on IMA by $0.71(95 \% \mathrm{Crl} 0.13$ to 1.32$)$.

Discussion. Resistance training not only improves strength, but also the structure of coordination in the control of a well-defined motor objective. The role of resistance training on motor abundance in gait should be investigated in patient cohorts, other gait patterns, and its translation into functional improvements. 
1 Article title: Not all Brawn, but some Brain. Strength gains after training alters kinematic motor 2 abundance in hopping.

\section{Authors}

Bernard X.W. Liew, BSc (Hon) ${ }^{1,4}$

Andrew Morrison, $\mathrm{PhD}^{2}$

Hiroaki Hobara, $\mathrm{PhD}^{3}$

Susan Morris, $\mathrm{PhD}^{4}$

Kevin Netto, $\mathrm{PhD}^{4}$

\section{Affiliations}

${ }^{1}$ Centre of Precision Rehabilitation for Spinal Pain (CPR Spine), School of Sport, Exercise and Rehabilitation Sciences, College of Life and Environmental Sciences, University of Birmingham, Birmingham, United Kingdom.

${ }^{2}$ Sport and Exercise Sciences, Faculty of Science and technology, Anglia Ruskin University, Cambridge, United Kingdom.

${ }^{3}$ Human Informatics Research Institute, National Institute of Advanced Industrial Science and Technology (AIST), Tokyo, Japan.

${ }^{4}$ School of Physiotherapy and Exercise Sciences, Curtin University, Perth, Western Australia, Australia.

\section{Address author}

3 Dr Bernard Liew, School of Sport, Exercise and Rehabilitation Sciences, Centre of Precision 4 Rehabilitation for Spinal Pain (CPR Spine), College of Life and Environmental Sciences, 5 University of Birmingham, Birmingham, United Kingdom; E-mail: LiewB@adf.bham.ac.uk;

6 liew_xwb@hotmail.com. Tel: +44 1214158526

Trial registration (Date of registration): ANZCTR (ACTRN12616000023459) (14 Jan 2016) 


\section{ABSTRACT.}

\section{Background.}

10 The effects of resistance training on a muscle's neural, architectural, and mechanical properties

11 are well established. However, whether resistance training can positively change the

12 coordination of multiple motor elements in the control of a well-defined lower limb motor performance objective remains unclear. Such knowledge is critical given that resistance training

14 is an essential and ubiquitous component in gait rehabilitation. This study aimed to investigate if

15 strength gains of the ankle and knee extensors after resistance training increases kinematic motor 16 abundance in hopping.

\section{Methods.}

18 The data presented in this study represents the pooled group results of a sub-study from a larger project investigating the effects of resistance training on load carriage running energetics. 30 healthy adults performed self-paced unilateral hopping, and strength testing before and after six weeks of lower limb resistance training. Motion capture was used to derive the elemental variables of planar segment angles of the foot, shank, thigh, and pelvis, and the performance variable of leg length. Uncontrolled manifold analysis (UCM) was used to provide an index of motor abundance (IMA) in the synergistic coordination of segment angles in the stabilization of

25 leg length. Bayesian Functional Data Analysis was used for statistical inference, with a non-zero crossing of the $95 \%$ Credible Interval (CrI) used as a test of significance.

\section{Results.}

Depending on the phase hop stance, there were significant main effects of ankle and knee strength on IMA, and a significant ankle by knee interaction effect. For example at $10 \%$ hop 
30 stance, a $1 \mathrm{Nm} / \mathrm{kg}$ increase in ankle extensor strength increased IMA by 0.37 (95\% CrI 0.14 to

$310.59)$, a $1 \mathrm{Nm} / \mathrm{kg}$ increase in knee extensor strength decreased IMA by 0.29 (95\% CrI 0.08 to

32 0.51), but increased the effect of ankle strength on IMA by 0.71 (95\% CrI 0.10 to 1.33$)$. At 55\%

33 hop stance, a $1 \mathrm{Nm} / \mathrm{kg}$ increase in knee extensor strength increase IMA by 0.24 (95\% CrI 0.001

34 to 0.48$)$, but reduced the effect of ankle strength on IMA by 0.71 (95\% CrI 0.13 to 1.32 ).

\section{Discussion.}

36 Resistance training not only improves strength, but also the structure of coordination in the

37 control of a well-defined motor objective. The role of resistance training on motor abundance in

38 gait should be investigated in patient cohorts, other gait patterns, and its translation into

39 functional improvements. 


\section{INTRODUCTION}

42 Regular participation in walking and running has important health benefits (Lee et al. 2014), and

43 is commonly undertaken along irregular surfaces. Normally, humans have no problems

44 maintaining dynamic postural control and energy efficiency during gait despite these surface

45 irregularities, ensuring a smooth center of mass (COM) trajectory. Excessive COM trajectory

46 disturbance in gait can be energetically costly and potentially destabilizing to postural control

47 (Andrada et al. 2013; Geyer et al. 2006).

Perturbation to the COM trajectory can be minimized over irregular surfaces by adjusting the length of a simplified virtual leg (henceforth termed as leg), spanning the COM to the center of pressure (COP) (Andrada et al. 2013; Geyer et al. 2006). Leg length is regulated by four major segments (foot, shank, thigh, and pelvis), along which flexion-extension occurs. The excess of segments required to control a single leg, means that the body has an abundance of solutions to flexibly combine segment angles to achieve the same leg length (Auyang et al. 2009). Greater motor abundance in leg length regulation affords the body greater adaptability to rapidly react to irregular surfaces to minimize COM trajectory perturbation. In the context of quantifying motor abundance in leg length regulation, the Uncontrolled Manifold (UCM) analysis has been used to investigate the motor control of unilateral hopping (Auyang et al. 2009). UCM provides a ratio of two variances: one where the variance in angles (motor elements) does not change leg length (performance variable) - Goal-Equivalent Variance (GEV), to a variance in angles which change leg length - Non Goal Equivalent Variance (NGEV) (Auyang et al. 2009).

61 The manifestation of normal abundance in motor task may depend on the task's physical demand 62 relative to an individual's physiological strength capacity (Greve et al. 2013; Olafsdottir et al. 2008; Park et al. 2015; Shim et al. 2008; Yen \& Chang 2010). Greater motor abundance may 
64 emerge when the task's relative physical demand increases (Greve et al. 2013). For example, older adults with lower maximal strength have non-significantly greater motor abundance in sitto-stand compared to younger adults with greater maximal strength (Greve et al. 2013). When an external load was added to walking, there was a significant increase in the motor abundance of joint angle co-variation in the control of the COM trajectory in the frontal plane, and a nonsignificant increase in abundance in the control of the COM trajectory in the sagittal plane $(\mathrm{Qu}$ 2012). It is reasonable to expect that if one muscle is operating near its physiological limit, additional muscles would be recruited to achieve successful performance.

In contrast, prospective resistance training studies of the upper limb demonstrated that greater wrist and finger strength was associated with greater abundance in finger force coordination tasks (Olafsdottir et al. 2008; Park et al. 2015; Shim et al. 2008). Resistance training may augment motor abundance via several mechanisms: (1) by increasing reciprocal inhibition of covarying muscle groups via heteronomous spinal pathways (Geertsen et al. 2008), (2) increasing the role of bi-articular muscles in inter-segmental kinematic co-variation (Cleather et al. 2015), and (3) increasing the number of muscle modes available for co-variation (Hashiguchi et al. 2016).

Although prospective study designs (Olafsdottir et al. 2008; Park et al. 2015; Shim et al. 2008) already provide a higher level of evidence base than a cross-sectional design (Greve et al. 2013; Qu 2012), the relationship between physiological strength and motor abundance may still have been confounded by other factors. First, different mathematical formulation of the variance ratios of motor abundance may have contributed to the conflicting evidence (Greve et al. 2013; Olafsdottir et al. 2008). For example, Olafsdottir et al. (2008) defined motor abundance using the ratio of $\frac{G E V-N G E V}{G E V+N G E V}$, whilst Greve et al. (2013) used a simple ratio of $\frac{G E V}{N G E V}$. Second, differences 
87 may lie in the hierarchical level of analysis as it pertains to the neural control of motor

88

89

90

91

92

93

94 abundance. Greve et al. (2013) investigated the covariation of joint-level kinematics to wholebody performance variables, such as ground reaction force. The analysis by Greve et al. (2013) thus did not consider an intermediary level of hierarchical control - that is in the stabilization of limb-level performance variables (Toney \& Chang 2016). In contrast, Olafsdottir et al. (2008) investigated the covariation of limb-level (finger) force in the stabilization of the total force generated by four fingers. To this end, a prospective study design that quantifies lower limb motor abundance within a hierarchical control framework would increase the evidence base behind using resistance training to improve motor abundance.

Resistance training is an essential component in gait rehabilitation (Papa et al. 2017). Whether a gain in physiological strength capacity benefits or harms motor abundance is an essential question to answer, as it directly implicates the role of resistance training in gait rehabilitation. The aim of this study was to investigate if lower limb strength gains after resistance training influenced lower limb motor abundance. Hopping represents an excellent model of forward gait patterns to fulfill the present study's aim. First, lower limb spring-mass dynamics in hopping is present in walking and running (Geyer et al. 2006). Second, leg length is a regulated performance variable in hopping using UCM analysis (Auyang et al. 2009). Given that leg length mechanics contributes to COM trajectory (Moritz \& Farley 2003), greater motor abundance in leg length stabilization in hopping may translate into more available motor solutions to minimize COM trajectory perturbation when walking or running over irregular surfaces. Third, normal inter-segmental kinematic and kinetic coordination in hopping, quantified using UCM and vector coding, varies depending on the task's relative physical demand (Auyang et al. 2009; Smith et al. 2014; Yen \& Chang 2010), Similar to the effects of finger muscle strengthening on finger 
110 pressing motor abundance (Olafsdottir et al. 2008), the hypothesis of this study was that greater

111 strength gains after resistance training would increase kinematic motor abundance in leg length

112 regulation during hopping.

\section{MATERIALS AND METHODS.}

\section{Participants}

115 The data presented in this manuscript represents the pooled group results of a sub-study from a

116 larger project investigating the effects of resistance training on load carriage running energetics

117 (Liew et al. 2017). (Liew et al. 2017). Healthy adult recreational runners between 18 to 60 years

118 old were invited to participate in the study. Participants had to be actively engaged in running or 119 running-related sports with a minimum cumulated total duration of 45 minutes per week to be 120 considered for inclusion. Exclusion criteria included: 1) self-reported medical conditions which

121 precluded the safe performance of running, jumping, hopping activities and heavy resistance

122 exercises; 2) self-reported running related injuries currently and within the past three months; 3)

123 surgeries within the past year; and 4) females who were pregnant at time of recruitment Thirty

124 participants volunteered for this study (16 male, 14 female). This study was approved by the

125 Curtin University Human Research Ethics Committee (RD-41-14). Informed written consent was 126 sought and gained prior to study enrolment.

\section{Intervention}

128 The two training programs were developed to improve load carriage running energetics (Table

129 S1 in supplementary material). One group performed "conventional" heavy-resistance isoinertial

130 training on the bilateral leg press, unilateral calf raises, and lunge exercises. These exercises have

131 been routinely adopted in conventional load-carriage military training (Knapik et al. 2012). The 
132 other group performed "load carriage specific" resistance training targeting the specific

133 biomechanical requirements of load carriage running (Liew et al. 2017). Exercises in this group

134 comprised of externally loaded single-leg hopping to increase leg stiffness, countermovement

135 jumps to increase knee power generation, and hip flexor pull to increase pre-swing running

136 energetics (Liew et al. 2017). Greater leg stiffness, knee power generation, and pre-swing hip

137 energetics were previously shown to be required to sustain constant running velocity during load

138 carriage (Liew et al. 2016a; Silder et al. 2015). Despite the differences between the training

139 programs, the present study was only interested in accounting for the between time (pre-post)

140 change in strength (ankle extensor $=$ mean increase $0.34 \mathrm{Nm} / \mathrm{kg}[95 \%$ Confidence Interval (CI)

1410.25 to $0.42 \mathrm{Nm} / \mathrm{kg}$ ]; $\mathrm{knee}$ extensor $=$ mean increase $0.24 \mathrm{Nm} / \mathrm{kg}$ [95\% CI 0.11 to $0.37 \mathrm{Nm} / \mathrm{kg}$ ])

142 (Liew et al. 2017), in predicting alterations in hopping motor abundance.

143 Three dimension motion capture on hopping (combined group analysis)

144 Participants performed unilateral hopping, on both sides, at a self-selected frequency lasting

145 approximately $15 \mathrm{~s}$. During hopping, the arms were held in a $90^{\circ}$ abducted position, to allow

146 visualization of the lateral pelvic markers. The only instruction provided was to hop at a

147 "comfortable pace". In the post hoc analysis stage, only hops maintained within $10 \%$ of the

148 individual's mean hop frequency was kept for further analysis (termed as successful trials). This

$14910 \%$ frequency window was deemed appropriate given that a previous study reported a variation

150 of up to $20 \%$ for adults hopping at their preferred frequency (Beerse \& Wu 2016). A between

151 side standing rest period of one minute was provided. An 18 camera motion capture system

152 (Vicon T-series, Oxford Metrics, UK) (250 Hz), with synchronized in-ground force plates

153 (AMTI, Watertown, MA) (2000 Hz) were used to collect marker trajectories and force data

154 (Vicon Nexus, v2.3, Oxford Metrics, UK). Force data were used to detect initial contact and toe- 
155 off, with a $20 \mathrm{~N}$ vertical force threshold used. The marker placements were based on a previous

156 study (Liew et al. 2016b). A seven-segment lower-limb biomechanical model was created in

157 Visual 3D (C-motion, Germantown, MD) (Liew et al. 2016a). Joint centers of the hip were

158 derived using a regression equation (Bell et al. 1989), whilst those of the knee and ankle were

159 derived as the midpoint between the medial and lateral femoral condyles, and malleoli,

160 respectively. Segment inertial and geometric properties were based on Visual 3D's default

161 routines. The biomechanical model's position and orientation was derived using inverse

162 kinematics. Each joint had three rotational degrees of freedom, with the model having a total of

16318 degrees of freedom. The laboratory and joint coordinate system used had the following

164 sequence: $\mathrm{X}$ axis - mediolateral with positive pointing to the right, $\mathrm{Y}$ axis - postero-anterior with

165 positive pointing anteriorly, and $\mathrm{Z}$ axis - vertical with positive pointing proximally. Marker

166 trajectories were low pass filtered at $12 \mathrm{~Hz}$ (zero lag, $4^{\text {th }}$ order, Butterworth).

\section{Isokinetic strength measurement}

168 Isokinetic concentric strength testing of the bilateral knee and ankle extensors was performed in 169 a dynamometer (HUMAC NORM, Computer Sports Medicine Inc., Stoughton, MA), collecting 170 data at $100 \mathrm{~Hz}$ and set up according to the manufacturer's guidelines (Liew et al. 2017). For each

171 muscle group tested, participants first performed 10 repetitions of warm-up contractions at $90^{\circ} / \mathrm{s}$,

172 and two sets of six maximal concentric-concentric contractions at $60^{\circ} / \mathrm{s}$. Each set was

173 interspersed with one minute of seated rest in-situ. Between muscle group and side rest periods

174 of three minutes were provided.

\section{Uncontrolled manifold analysis}

176 A modified sagittal plane forward kinematic model mapping segment angles to leg length used in

177 a previous study was adopted in the present study (Auyang et al. 2009) (Figure 1). Leg length 
178 was presently defined by the vector between the centre of pressure (COP) to the proximal end of

179 the pelvic segment, instead of the toe and anterior superior iliac spine markers, respectively

180 (Auyang et al. 2009). The X-coordinate of landmarks used to create the planar segments was set

181 to zero. The foot, shank, thigh, and pelvic planar segments were defined by the line vectors in the

182 YZ plane between 1) COP to ankle joint centre, 2) ankle to knee joint centre, 3) knee to hip joint

183 centre, and 4) hip joint to proximal end of the pelvic inertial segment. Trial-to-trial variability in

184 leg length can be influenced by variable changes to segment lengths, given the presence of soft

185 tissue artefact. This effect was minimized by using landmarks modeled after the biomechanical

186 model was optimized using inverse kinematic. Sagittal planar angles of each segment in the YZ

187 plane were defined relative to the laboratory's horizontal plane, using the Right Hand Rule. All

188 segment planar angles and leg length were time-normalized to 100 data points in the stance

189 period for UCM analysis.

190 The UCM analysis was carried out using a previously published method (Auyang et al. 2009),

191 for each of the 100 stance data points.

192

193

194

$$
N G E V=\frac{\operatorname{trace}\left(\operatorname{orth}\left(J^{t}\right)^{t} \cdot \operatorname{Corth}\left(J^{t}\right)\right)}{d}
$$

$$
G E V=\frac{\operatorname{trace}\left(\operatorname{null}(J)^{t} \cdot \operatorname{Corth}(J)\right)}{n-d} \ldots \text { (2) }
$$

$$
\mathrm{IMA}=\frac{G E V-N G E V}{G E V+N G E V} \ldots
$$

In equations (1) to (3), non-goal equivalent variance (NGEV) represented the variance of all segment angle combinations that contributed to leg length changes while goal equivalent variance $(\mathrm{GEV})$ represented the variance of all segment angle combinations that did not change 
198 leg length. The index of motor abundance (IMA) represented the ratio of two variance measures

199 (Auyang et al. 2009). Thus an IMA $>0$ characterized variation in segment angles which

200 minimized leg length variation (i.e. motor abundance), and an IMA $<0$ characterized variation in

201 segment angles that maximized leg length variation (Auyang et al. 2009). In the equation, $\mathrm{J}$ is the

202 Jacobian matrix mapping infinitesimally small changes in segment angles to changes in leg

203 length; $\mathrm{C}$ is the co-variance matrix in the deviation of the segment angles from the mean

204 reference segment angles at each datum; $d$ is the degree of freedom in the performance variable

$205(d=1$ in this study); and $n$ is the degree of freedom in the elemental variables $(n=4)$.

\section{Statistical analysis (combined group inference)}

207 A previous study reported that the standard deviation of the GEV and NGEV was lowest with at least 20 trials (Latash et al. 2010). Hence, UCM analysis and subsequent functional regression analyses was performed only on participants with $\geq 20$ successful trials. Simple linear regression was used to quantify differences in age, height, weight, running frequency (times/week) and cumulated distance $(\mathrm{km} /$ week) over the past six weeks, baseline ankle and knee extensor strength between participants with and without $\geq 20$ successful trials.

Descriptive scalar variables of post-pre change in hopping frequency and stance duration, and baseline (pre-training) waveform variables of leg length, foot, shank, thigh, and pelvic segment angles, IMA, GEV, and NGEV were reported for participants with $\geq 20$ successful hopping trials. The dependent variable was the between time change in waveform IMA. The predictor variables were the between time change (post-pre) in ankle, knee strength and their interaction, and the regression coefficients were adjusted for three covariates: 1) change (post-pre) in hopping frequency, 2) side (right vs left); and (3) total number (post + pre) of hopping trials were included in the statistical model. These statistical adjustments were made given that changes in 
221 IMA between pre- and post-testing could be due to (1) changes in hopping frequency, (2) limb

222 dominance, and (3) the number of trials used for UCM analysis; and we wish to isolate the

223 estimate of strength gains on IMA changes. Bayesian regression functional analysis was

224 performed in R software (Goldsmith \& Kitago 2016). Recent investigations in sports science

225 have advocated the avoidance of frequentist null-hypothesis significance testing, and instead to

226 focus on estimating the probabilities associated with observing an effect size. Fixed effect

227 parameters for ankle and knee strength, frequency, side, trial number, and non-parametric

228 smooth functions (modelled with 15 B-splines) were estimated using a Gibbs sampler with a

229 burn-in of 1000 and drawing 15000 inference samples. The residual covariance structure was

230 estimated using Bayesian functional principle components. A significant effect was defined by a

231 non-zero crossing of the Bayesian 95\% credible interval (CrI).

\section{RESULTS}

233 Twenty-five participants had $\geq 20$ successful trials (Figure 2). No significant differences in

234 baseline characteristics between participants with and without $\geq 20$ successful trials, were

235 detected (Table 1). For the 25 participants with $\geq 20$ successful trials, the number of hop trials

236 used for UCM analysis per participant ranged from 20 to 57.

237 Baseline hopping kinematics, IMA, GEV, NGEV are reported in Figure 3 and 4. For the 25

238 participants with $\geq 20$ successful trials, the mean (standard deviation) change in hopping

239 frequency was a $0.15(0.25) \mathrm{Hz}$ increase, and change in stance duration was a $0.01(0.03) \mathrm{s}$

240 decrease post-testing, relative to a baseline of $2.24(0.26) \mathrm{Hz}$ and $0.31(0.04) \mathrm{s}$, respectively.

241 For simplicity, only effects at a discrete hop phase within a statistically significant temporal

242 period are reported here. At $10 \%$ hop stance, there was a significant interaction between ankle 
243 and knee strength gains, and significant main effect of ankle and knee strength gains (Figure 5).

244 A $1 \mathrm{Nm} / \mathrm{kg}$ increase in ankle extensor strength increased IMA by 0.37 (95\% CrI 0.14 to 0.59 ), a

$2451 \mathrm{Nm} / \mathrm{kg}$ increase in knee extensor strength decreased IMA by 0.29 (95\% CrI 0.08 to 0.51$)$, but

246 increased the effect of ankle strength on IMA by 0.71 (95\% CrI 0.10 to 1.33 ) (Figure 5). At 55\%

247 hop stance, a $1 \mathrm{Nm} / \mathrm{kg}$ increase in knee extensor strength increase IMA by 0.24 (95\% CrI 0.001

248 to 0.48$)$, but reduced the effect of ankle strength on IMA by 0.71 (95\% CrI 0.13 to 1.32 ) (Figure

249 5). At $70 \%$ hop stance, a $1 \mathrm{Nm} / \mathrm{kg}$ increase in ankle extensor strength reduced IMA by 0.31

250 (95\% CrI 0.06 to 0.58 ) (Figure 5). At $98 \%$ hop stance, a $1 \mathrm{Nm} / \mathrm{kg}$ increase in ankle extensor

251 strength increased IMA by 0.39 (95\% CrI 0.05 to 0.73$)$ (Figure 5).

\section{DISCUSSION}

253 Leg length regulation is a strategy of coping with irregular surfaces to minimize disturbance to

254 the COM trajectory during gait (Andrada et al. 2013; Geyer et al. 2006). Normally, leg length

255 regulation is achieved by harnessing segmental kinematic motor abundance (Auyang et al. 2009).

256 Even though resistance training has been typically prescribed to treat gait impairments, there is

257 uncertainty as to the relationship between physiological strength capacity and normal motor

258 abundance. This poses a dilemma as to whether resistance training benefits or harms gait motor

259 control rehabilitation. In this study, we prospectively investigated if lower limb strength gains

260 after resistance training, predicted a change in IMA during a simple model of spring-mass gait -

261 unilateral hopping. In partial agreement with our hypothesis, greater strength gains predicted an

262 increase in IMA, but this effect was dependent on the muscles being strengthened. In addition,

263 the effects of ankle strength gained on IMA was opposite to that of knee strength gains. 
264 The increase in kinematic motor abundance with an isolated gain in ankle extensor strength after

265 initial contact and toe-off of hopping, was consistent with the findings of previous resistance

266 training studies of the upper limb (Olafsdottir et al. 2008; Park et al. 2015; Shim et al. 2008). The

267 mechanisms linking strength gains to motor abundance improvements was not investigated in the

268 present study, but may speculatively involve mechanical and neural factors. The bi-articular

269 gastrocnemius is mechanically capable of plantar flexing the foot while flexing the knee

270 (Cleather et al. 2015). Greater gastrocnemius strength could augment inter-segmental kinematic

271 co-variation, increasing motor abundance during hopping. Resistance training has also been

272 shown to increase reciprocal inhibition of antagonistic muscles within a joint muscle pair

273 (Geertsen et al. 2008). However, no studies to the authors' knowledge have directly investigated

274 the influence of resistance training on heteronomous reflex pathways, which would enable inter-

275 muscular co-variation and facilitate hopping kinematic motor abundance.

276 It was previously suggested that greater motor abundance will emerge in tasks with greater

277 relative physical demand (Greve et al. 2017; Greve et al. 2013), which contradicts the present

278 findings. It may be that the relationship between physical demand and motor abundance is non-

279 linear with potentially plateauing effects. Both physiological weakening and strengthening may

280 augment motor abundance depending in part on the task's absolute demand on the participants,

281 and the capacity to use available motor elements. Wang et al. reported that older adults have

282 preserved muscle motor abundance but were more delayed at their recruitment during rapid

283 balance recovery, than younger adults (Wang et al. 2017). The tasks used by Greve and

284 Colleagues were either much slower, or required a combination of low muscular force and fast

285 movement speed (Greve et al. 2017; Greve et al. 2013). It is plausible that the speed-force

286 demands in previous studies were low (Greve et al. 2017; Greve et al. 2013), such that muscle 
287 groups had sufficient time to stabilize the performance variable(s). In addition, if a muscle is

288 operating at its physiological limit, it can no longer compensate for the reduction in activation of

289 other muscles. In this instance, co-variation may still occur but only between muscles with

290 adequate physiological strength reserve. The relationship between task demand and motor

291 abundance may be better understood by investigating abundance at the level of muscle

292 activations, including UCM analysis in a reduced subset of motor elements (Toney \& Chang 293 2016).

294 The period surrounding mid-stance in hopping is critical of leg length regulation for peak

295 muscular force minimization (Auyang et al. 2009), which minimizes the energy expenditure and

296 joint contact loads during hopping. If knee strength gain occurred in isolation, an increase in

297 motor abundance at 55\% stance was observed. However, if strength gains occurred at the ankle

298 and knee, due to the statistical interaction, motor abundance was reduced. The detrimental effect

299 of additional ankle strength gain could be due to the foot segment angle around mid-stance being

300 nearly invariant (Figure 3) (Joao et al. 2014). If the foot functions as a punctum fixum around

301 mid-stance (Joao et al. 2014), the gastrocnemius is only able to flex and extend the thigh

302 segment, without compensatory foot kinematics to stabilize overall leg length. The invariant

303 foot-segment angle may instead shift the joint-level mediator of leg length stabilization to the

304 knee during the period of mid-stance.

305 Greater knee extensor strength reduced motor abundance after initial contact, but augmented the 306 incremental effect ankle strength gain had on abundance for leg length control. It may be that the 307 influence of knee extensor strength gain on motor abundance was shifted to the control of leg 308 orientation (angle between the leg and ground) (Auyang et al. 2009). Leg orientation at initial 
309 contact may be critical as it determines the overall position of the force application relative to the

$310 \mathrm{COM}$ in stance.

311 The reduction in kinematic motor abundance predicted by an increase in knee extensor strength

312 after initial contact differed from a study investigating walking in individuals with and without

313 knee osteoarthritis (OA) (Tawy et al. 2018). Several reasons could account for the disagreement.

314 Tawy et al. (2018) did not directly quantify the relationship between knee extensor strength and

315 motor abundance. The occurrence of knee OA is associated with a range of neuromuscular

316 deficits (Mills et al. 2013), and the importance of knee extensor strength to motor abundance

317 cannot be ascertained from a between-groups comparison. Second, Tawy et al. (2018) used COM

318 trajectory, while the present study used leg length, as the performance variable for UCM

319 analysis. It must be emphasized that using both the COM and leg length as performance

320 variables are equally valid. The organization of motor control may involve a hierarchical

321 structure (Latash 2010), where segment-level variation serve to stabilize limb-level outcomes,

322 and inter-limb variation stabilizes whole-body outcomes. Thus, the present study focused only on

323 limb-level motor control, while Tawy et al. (2018) performed UCM analysis across two layers of

324 hierarchical control.

325 Several aspects of the present study's methodology need to be discussed in lieu of differences in 326 reported IMA of the present study, with that of a previous work (Auyang et al. 2009). First, the

327 number of hop cycles included in the present study was much lower than the 170 cycled used in

328 Auyang et al. (2009). This may explain the difference in IMA values between studies. Second,

329 leg length was defined starting from the COP in the present study, but from the toe marker in

330 Auyang et al. (2009). COP accuracy may be reduced when the magnitude of the GRF is small,

331 which could explain the differences in IMA between the present study and Auyang et al. (2009) 
332 during the periods surrounding initial contact and toe-off. However, the effective leg length

333 during human locomotion may be more accurately defined from the point of ground force

334 application, compared to the fixed toe-marker (Coleman et al. 2012). Despite this difference in

335 leg length definition, the overall shape of the IMA reported in this study was similar to Auyang 336 et al. (2009).

337 Previous studies provided evidence for the benefit of resistance training on finger force motor 338 abundance (Olafsdottir et al. 2008; Park et al. 2015; Shim et al. 2008), and the results of the 339 present study extends the evidence for the same benefit to the lower limb. Findings from the 340 present study carry an optimistic message that strength training may benefit the rehabilitation of 341 gait where movement coordination and strength are impacted upon by the presence of disease 342 (Hashiguchi et al. 2016). By increasing motor abundance to stabilize leg length in hopping, 343 resistance training may increase the adaptability of forward gait patterns over irregular surfaces.

344 It is likely that different gait patterns require different joint-level and limb-level strengthening to

345 benefit kinematic motor abundance, and this should be investigated in future studies. The present 346 study's findings also demonstrate that local strength changes can influence movement

347 coordination across the kinematic chain. Speculatively, this may imply that where strength gains 348 cannot be feasibly achieved using a more functional form of strength training, a more regionally 349 focused form of training (e.g. open-kinetic chain exercises) can still have global functional 350 benefits. Whether different strength training modes differentially influence lower limb motor 351 abundance, remains to be investigated.

352 A limitation of this study was that the analysis predicting motor abundance from alterations in 353 strength gains were analyzed using a prospective, pre-post design. However, we reduced the 354 confounding factor of repeated measurement, by only including the effects of strength changes 
355 into the statistical model. A second limitation of this study was that the influence of strength

356 gains on kinematic abundance was analyzed in healthy individuals. This limitation may in fact be

357 a strength, as we were able to isolate the investigation of IMA changes to strength changes.

\section{CONCLUSIONS}

359

360

361

362

363

364

365

366

367

368

369

370

371

372

373

374

375

376

377

In addition to the well-known effects on a muscle's neural, architectural, and mechanical properties, resistance training also influences the coordination of multiple motor elements in the control of a well-defined motor performance objective. The benefits of strength gain on motor abundance was dependent on the site of muscle strengthened and the phase of gait. The role of resistance training on motor abundance should be investigated in patient cohorts, other gait patterns, as well as its translation into functional improvements.

\section{ACKNOWLEDGEMENTS.}

The authors of this study would like to thank Nour Faiz Aqil Yaccob, Jason Hu, Nicholas Callaghan, Tess Moynihan, Hannah Watt, and Giorgia Alford for delivering the interventions. The results of this study are presented clearly, honestly, and without fabrication, falsification, or inappropriate data manipulation.

\section{References}

Andrada E, Nyakatura JA, Bergmann F, and Blickhan R. 2013. Adjustments of global and local hindlimb properties during terrestrial locomotion of the common quail $(<\mathrm{em}>$ Coturnix coturnix </em>). The Journal of Experimental Biology 216:3906-3916. $10.1242 /$ jeb.085399

Auyang AG, Yen JT, and Chang YH. 2009. Neuromechanical stabilization of leg length and orientation through interjoint compensation during human hopping. Experimental Brain Research 192:253-264. 10.1007/s00221-008-1582-7 
378 Beerse M, and Wu J. 2016. Vertical stiffness and center-of-mass movement in children and

379

380

381

382

383

384

385

386

387

388

389

390

391

392

393

394

395

396

397

398

399

400 adults during single-leg hopping. Journal of Biomechanics 49:3306-3312. http://dx.doi.org/10.1016/j.jbiomech.2016.08.014

Bell AL, Brand RA, and Pedersen DR. 1989. Prediction of hip joint centre location from external landmarks. Human Movement Science 8:3-16. http://dx.doi.org/10.1016/0167$\underline{9457(89) 90020-1}$

Cleather DJ, Southgate DFL, and Bull AMJ. 2015. The role of the biarticular hamstrings and gastrocnemius muscles in closed chain lower limb extension. Journal of Theoretical Biology 365:217-225. https://doi.org/10.1016/j.jtbi.2014.10.020

Coleman DR, Cannavan D, Horne S, and Blazevich AJ. 2012. Leg stiffness in human running: Comparison of estimates derived from previously published models to direct kinematickinetic measures. Journal of Biomechanics 45:1987-1991.

10.1016/j.jbiomech.2012.05.010

Geertsen SS, Lundbye-Jensen J, and Nielsen JB. 2008. Increased central facilitation of antagonist reciprocal inhibition at the onset of dorsiflexion following explosive strength training. Journal of applied physiology (Bethesda, Md : 1985) 105:915-922. 10.1152/japplphysiol.01155.2007

Geyer H, Seyfarth A, and Blickhan R. 2006. Compliant leg behaviour explains basic dynamics of walking and running. Proceedings of the Royal Society of London Series B - Biological Sciences 273:2861-2867. 10.1098/rspb.2006.3637

Goldsmith J, and Kitago T. 2016. Assessing systematic effects of stroke on motorcontrol by using hierarchical function-on-scalar regression. Journal of the Royal Statistical Society Series C, Applied statistics 65:215-236. 10.1111/rssc.12115 
401 Greve C, Hortobagyi T, and Bongers RM. 2017. Old adults preserve motor flexibility during

402

403

404

405

406

407

408

409

410

411

412

413

414

415

416

417

418

419

420

421

422

423 rapid reaching. European Journal of Applied Physiology 117:955-967. 10.1007/s00421017-3584-2

Greve C, Zijlstra W, Hortobagyi T, and Bongers RM. 2013. Not all is lost: old adults retain flexibility in motor behaviour during sit-to-stand. PLoS ONE [Electronic Resource] 8:e77760. 10.1371/journal.pone.0077760

Hashiguchi Y, Ohata K, Kitatani R, Yamakami N, Sakuma K, Osako S, Aga Y, Watanabe A, and Yamada S. 2016. Merging and Fractionation of Muscle Synergy Indicate the Recovery Process in Patients with Hemiplegia: The First Study of Patients after Subacute Stroke. Neural Plasticity 2016:5282957. 10.1155/2016/5282957

Joao F, Veloso A, Cabral S, Moniz-Pereira V, and Kepple T. 2014. Synergistic interaction between ankle and knee during hopping revealed through induced acceleration analysis. Human Movement Science 33:312-320. 10.1016/j.humov.2013.10.004

Knapik JJ, Harman EA, Steelman RA, and Graham BS. 2012. A systematic review of the effects of physical training on load carriage performance. Journal of Strength \& Conditioning Research 26:585-597. 10.1519/JSC.0b013e3182429853

Latash ML. 2010. Motor Synergies and the Equilibrium-Point Hypothesis. Motor Control 14:294-322.

Latash ML, Levin MF, Scholz JP, and Schöner G. 2010. Motor Control Theories and Their Applications. Medicina (Kaunas) 46:382-392.

Lee DC, Pate RR, Lavie CJ, Sui X, Church TS, and Blair SN. 2014. Leisure-time running reduces all-cause and cardiovascular mortality risk. Journal of the American College of Cardiology 64:472-481. 10.1016/j.jacc.2014.04.058 
424 Liew B, Morris S, and Netto K. 2017. The biomechanics of running with load PhD. Curtin $425 \quad$ University; .

426 Liew BX, Morris S, and Netto K. 2016a. Joint power and kinematics coordination in load 427 carriage running: Implications for performance and injury. Gait \& Posture 47:74-79.

Liew BX, Morris S, Robinson MA, and Netto K. 2016b. Performance of a lateral pelvic cluster technical system in evaluating running kinematics. Journal of Biomechanics. 10.1016/j.jbiomech.2016.05.010

Mills K, Hunt MA, Leigh R, and Ferber R. 2013. A systematic review and meta-analysis of lower limb neuromuscular alterations associated with knee osteoarthritis during level walking. Clin Biomech (Bristol, Avon) 28:713-724. 10.1016/j.clinbiomech.2013.07.008

Moritz CT, and Farley CT. 2003. Human hopping on damped surfaces: strategies for adjusting leg mechanics. Proceedings of the Royal Society of London Series B - Biological Sciences 270:1741-1746. 10.1098/rspb.2003.2435

Olafsdottir HB, Zatsiorsky VM, and Latash ML. 2008. The effects of strength training on finger strength and hand dexterity in healthy elderly individuals. Journal of applied physiology (Bethesda, Md : 1985) 105:1166-1178. 10.1152/japplphysiol.00054.2008

Papa EV, Dong X, and Hassan M. 2017. Resistance training for activity limitations in older adults with skeletal muscle function deficits: a systematic review. Clinical interventions in aging 12:955-961. 10.2147/cia.s104674

Park J, Han D-W, and Shim JK. 2015. Effect of Resistance Training of the Wrist Joint Muscles on Multi-Digit Coordination. Perceptual and Motor Skills 120:816-840. 10.2466/25.26.PMS.120v16x9

Qu X. 2012. Uncontrolled manifold analysis of gait variability: effects of load carriage and fatigue. Gait Posture 36:325-329. 10.1016/j.gaitpost.2012.03.004 
448 Shim JK, Hsu J, Karol S, and Hurley BF. 2008. Strength training increases training-specific 449 multifinger coordination in humans. Motor Control 12:311-329.

450

451

452 453

454

455

456 457

458

459 460

461

462

463

464

465

466

467

468

Silder A, Besier T, and Delp SL. 2015. Running with a load increases leg stiffness. Journal of Biomechanics 48:1003-1008. 10.1016/j.jbiomech.2015.01.051

Smith JA, Popovich JM, Jr., and Kulig K. 2014. The influence of hip strength on lower-limb, pelvis, and trunk kinematics and coordination patterns during walking and hopping in healthy women. Journal of Orthopaedic \& Sports Physical Therapy 44:525-531. 10.2519/jospt.2014.5028

Tawy GF, Rowe P, and Biant L. 2018. Gait variability and motor control in patients with knee osteoarthritis as measured by the uncontrolled manifold technique. Gait \& Posture 59:272-277. https://doi.org/10.1016/j.gaitpost.2017.08.015

Toney ME, and Chang YH. 2016. The motor and the brake of the trailing leg in human walking: leg force control through ankle modulation and knee covariance. Experimental Brain Research 234:3011-3023. 10.1007/s00221-016-4703-8

Wang Y, Watanabe K, and Asaka T. 2017. Aging effect on muscle synergies in stepping forth during a forward perturbation. European Journal of Applied Physiology 117:201-211. $10.1007 / \mathrm{s} 00421-016-3514-8$

Yen JT, and Chang YH. 2010. Rate-dependent control strategies stabilize limb forces during human locomotion. Journal of the Royal Society, Interface 7:801-810.

10.1098/rsif.2009.0296 
Figure 1

Planar kinematic model 


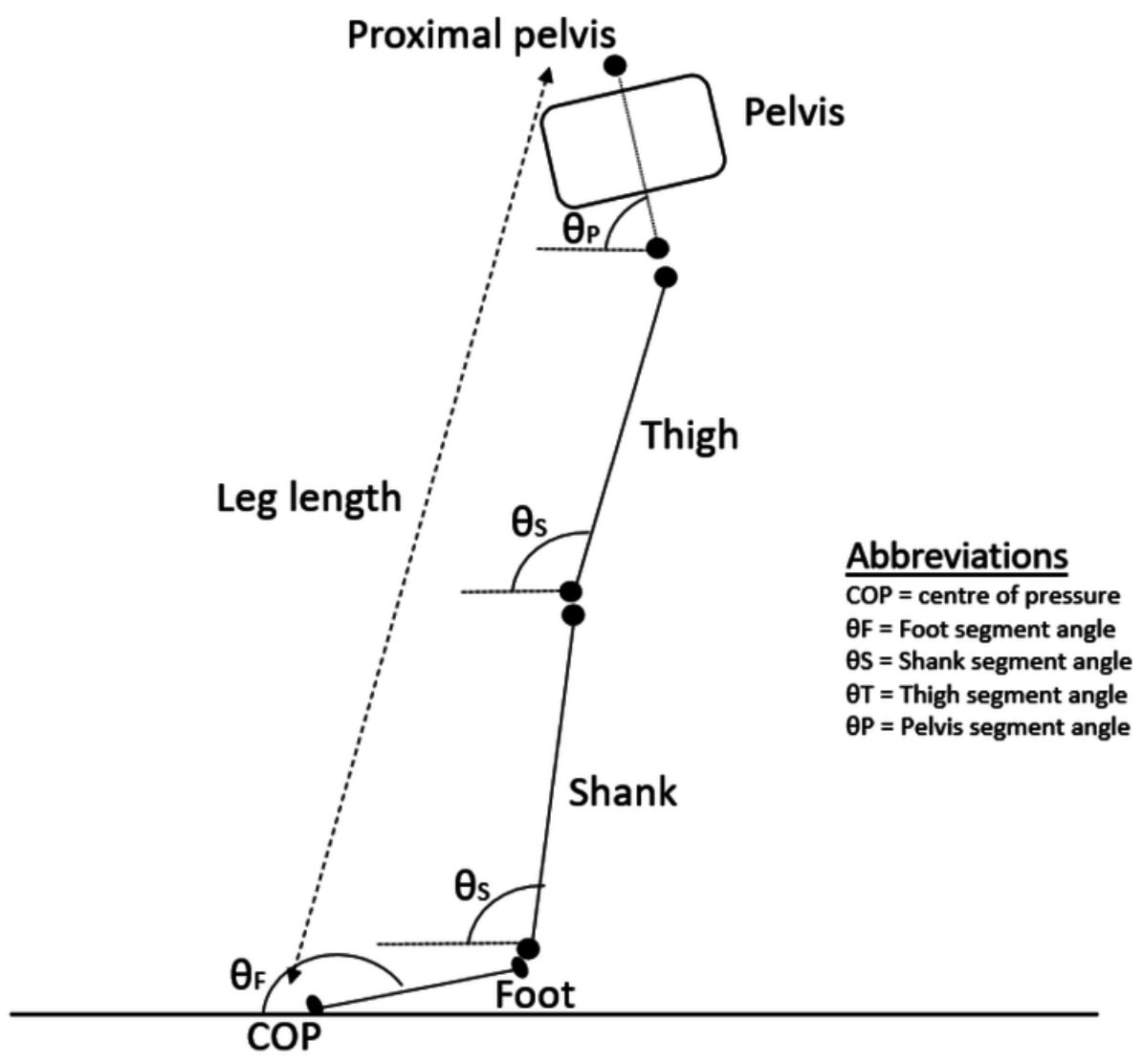


Figure 2 (on next page)

CONSORT Diagram 


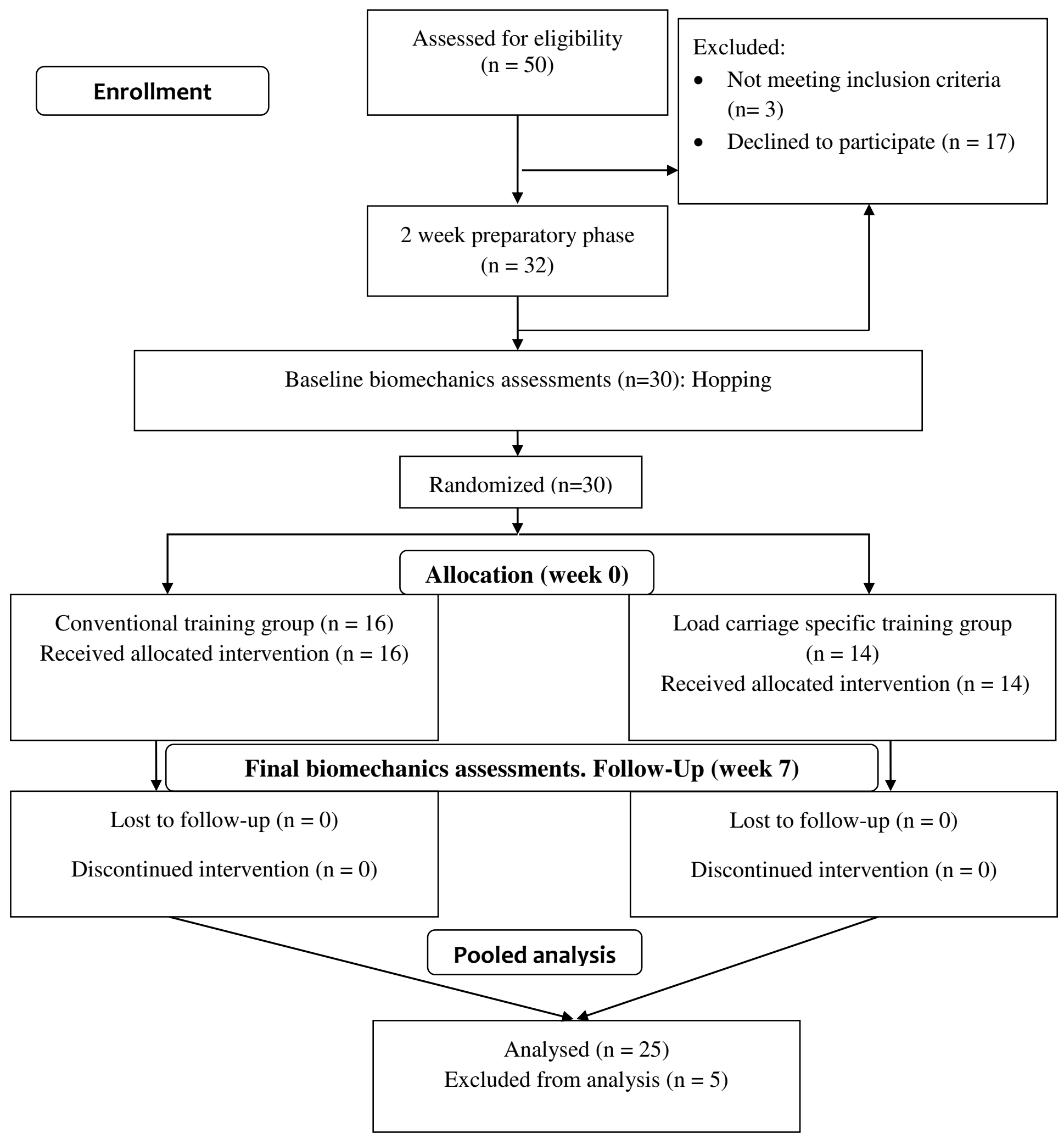


Figure 3

Baseline mean (standard deviation as error clouds) of leg length and segment angles

A

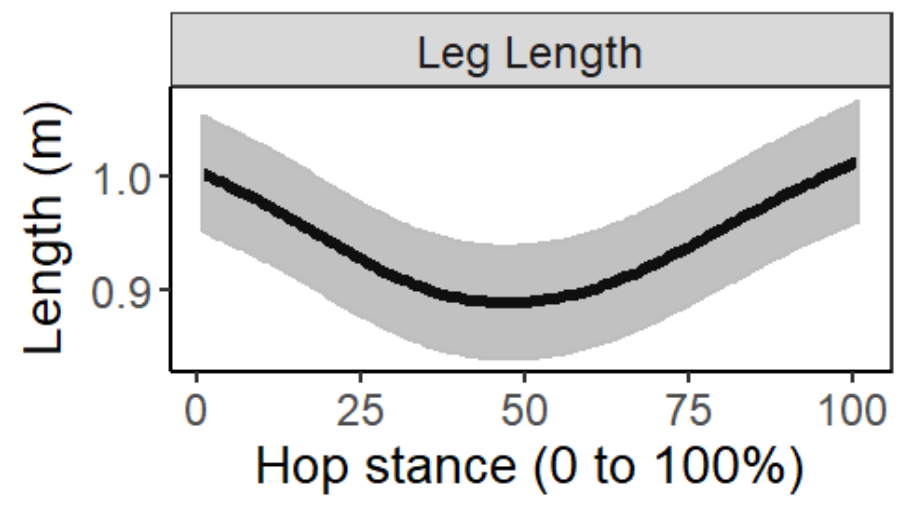

C

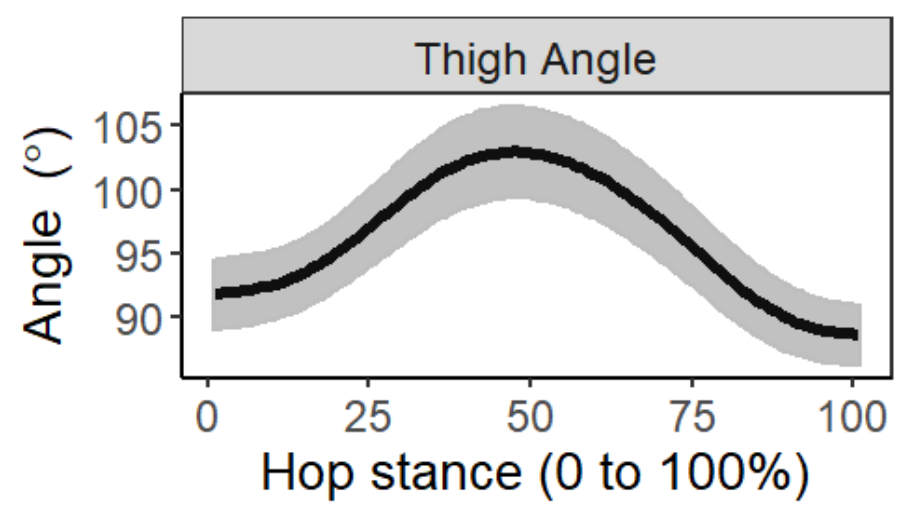

$\mathrm{E}$

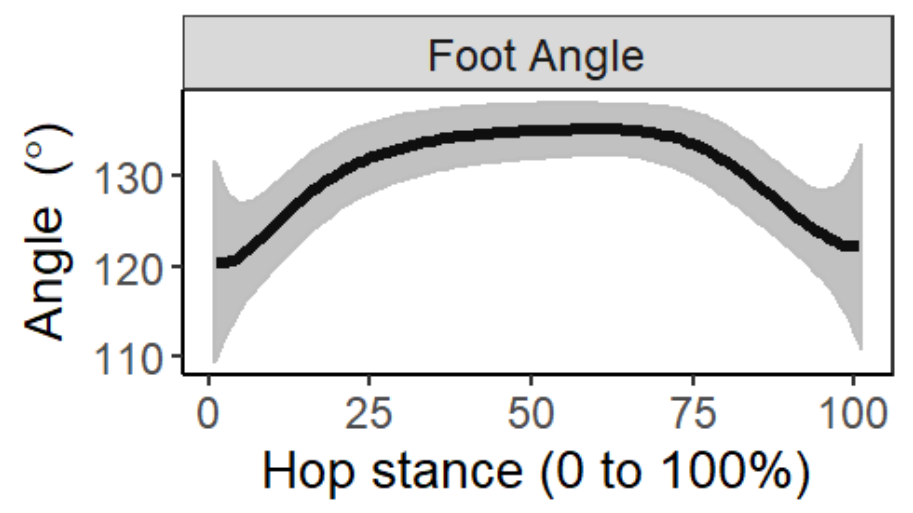

B

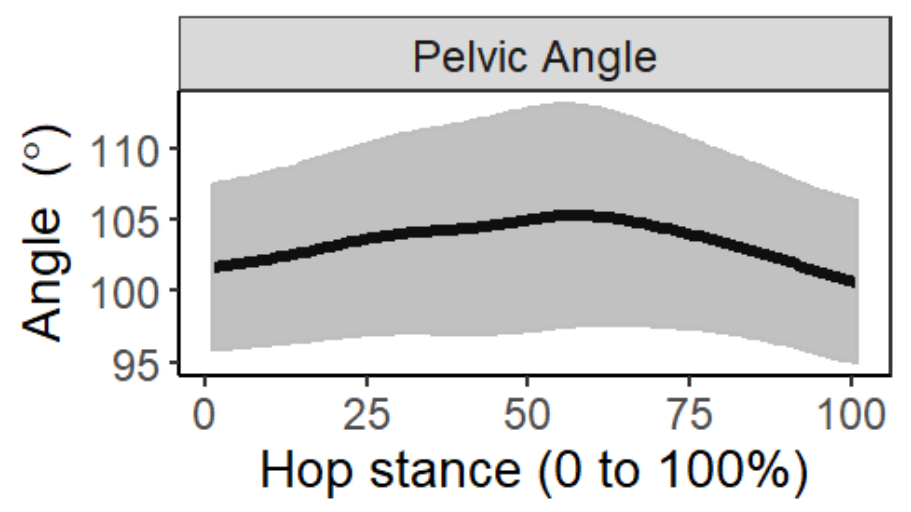

$\mathrm{D}$

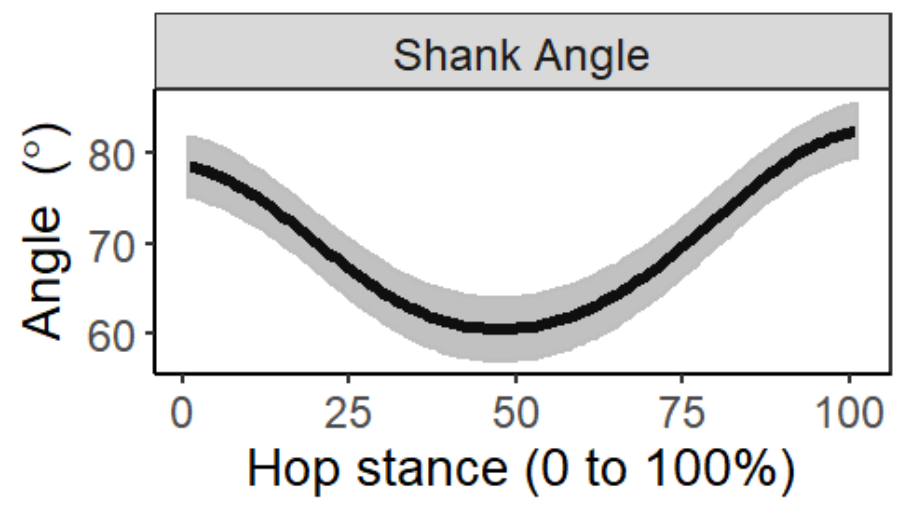


Figure 4

Baseline mean (standard deviation as error clouds) of Index of Motor Abundance (IMA), Non-Goal Equivalent Variance (NGEV) and Goal Equivalent Variance (GEV)
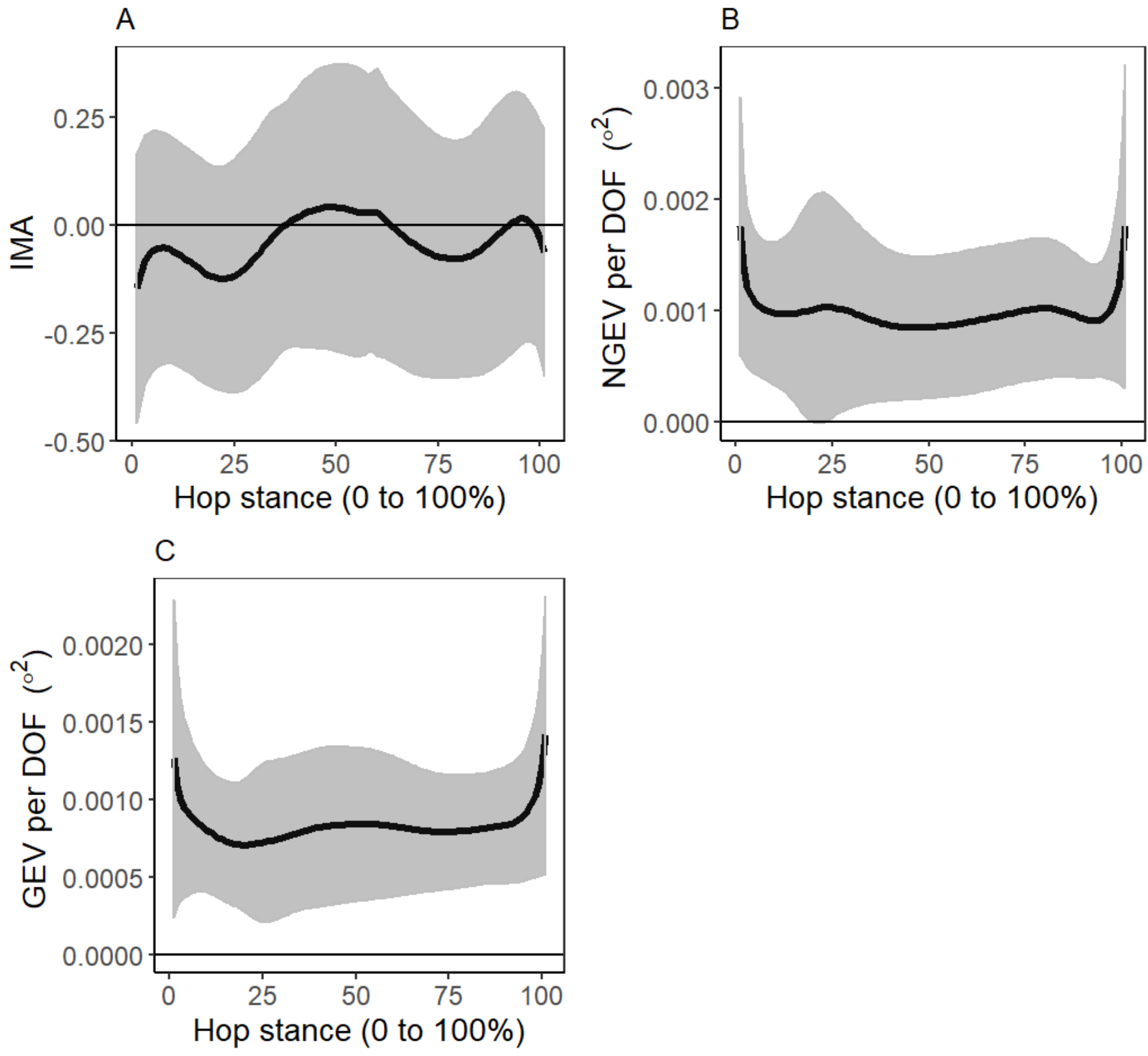
Figure 5

Mean (95\% Credible Interval as error clouds) of beta coefficient of $1 \mathrm{Nm} / \mathrm{kg}$ increase in ankle extensor strength (A) and knee extensor strength (B), and its interaction (C).
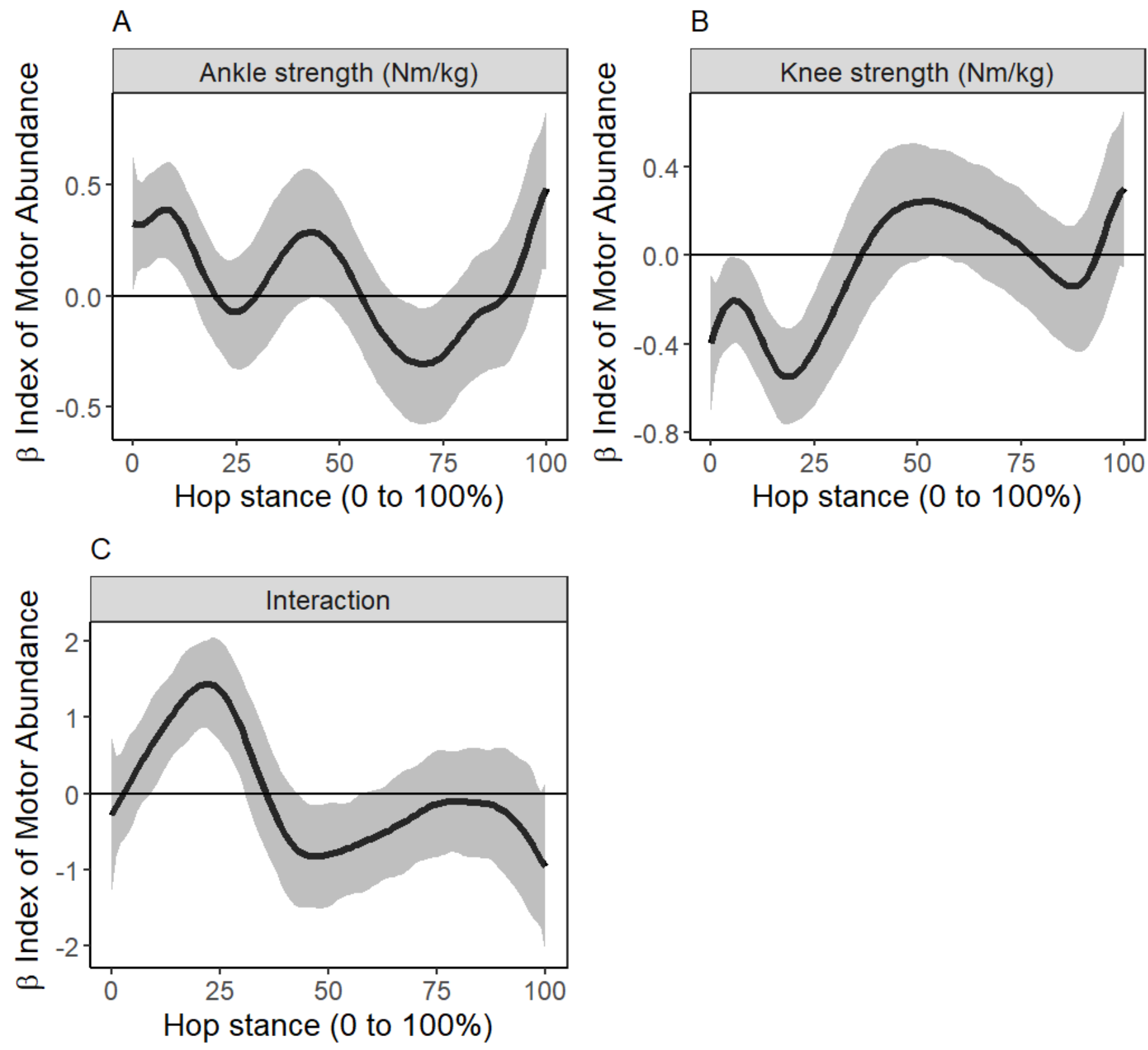
Table $\mathbf{1}$ (on next page)

Baseline characteristics of participants 
1 Table 1

\begin{tabular}{|l|l|l|l|}
\hline & $\begin{array}{l}\geq 20 \text { hop trials for } \\
\text { UCM } \\
(\mathrm{n}=25)\end{array}$ & $\begin{array}{l}<20 \text { hop trials for } \\
\text { UCM } \\
(\mathrm{n}=5)\end{array}$ & p value \\
\hline Age (years) & $30.5(9.7)$ & $28.6(6.5)$ & 0.684 \\
\hline Body mass $(\mathrm{kg})$ & $67.1(12.2)$ & $74.9(11.1)$ & 0.196 \\
\hline Height (cm) & $171.3(7.6)$ & $176.1(7.5)$ & 0.210 \\
\hline $\begin{array}{l}\text { Running frequency } \\
\text { over past 6 weeks } \\
\text { (times/week) }\end{array}$ & $2.7(1.4)$ & $2.2(1.3)$ & 0.423 \\
\hline $\begin{array}{l}\text { Running distance } \\
\text { over past 6 weeks } \\
\text { (km/week) }\end{array}$ & $16.8(18.8)$ & $15.2(10.1)$ & 0.852 \\
\hline $\begin{array}{l}\text { Ankle strength } \\
\text { (Nm/kg) }\end{array}$ & $1.07(0.19)$ & $1.07(0.24)$ & 0.978 \\
\hline $\begin{array}{l}\text { Knee strength } \\
\text { (Nm/kg) }\end{array}$ & $2.05(0.39)$ & $2.09(0.42)$ & 0.786 \\
\hline
\end{tabular}

2 